\title{
Mouse Pointer Movement using Gaze Tracking System
}

\author{
Shubham \\ Zende \\ Computer \\ Engineering \\ Vishwakarma \\ Institute of \\ Information \\ Technology Pune- \\ 48
}

\author{
Vinaya \\ Tambile \\ Computer \\ Engineering \\ Vishwakarma \\ Institute of \\ Information \\ Technology Pune- \\ 48
}

\author{
Akash Thakur \\ Computer \\ Engineering \\ Vishwakarma \\ Institute of \\ Information \\ Technology Pune- \\ 48
}

\author{
Mahesh \\ Shendge \\ Computer \\ Engineering \\ Vishwakarma \\ Institute of \\ Information \\ Technology Pune- \\ 48
}

\author{
Snehal Rathi \\ Computer \\ Engineering \\ Vishwakarma \\ Institute of \\ Information \\ Technology Pune- \\ 48
}

\begin{abstract}
The Gaze tracking system has been developed to provide access of computer for people with disabilities. Low cost and low complexity technique is used in our technique. In these system we use hardware such as Eye Touch Goggles and micro controller. Micro controller is based on data acquisition (DAQ) system. This system will be capable of performing left click, right click, and double clicks using appropriate eye winks of the user. In addition to that we have also tried to perform some tasks like open or close folder, reading pdf file. In this system we are using Infra-red sensors (IR sensors) for building light-reflection based system with non-imaging sensors like photo-diodes. This system is basically low Complexity system. IR sensors give us accurate approximation of the point of eye gaze. A system can be developed that would listen to DAQ device and performs major tasks like providing a user interface, determining current action performed by user and accordingly performing the action related to the current event. And hence cursor movement can be done. This proposed system will provide a promising interface between computer and human.
\end{abstract}

\section{Keywords}

HCI, gaze estimation, eyeball tracking, blink detection.

\section{INTRODUCTION}

Eye gaze tracking system has wide functional operations like human-machine interface, virtual reality, and diagnosis of diseases related to eyes. In real world this system will help disable people to operate and control the computer systems effectively. The Handicapped people face lot many problems in interacting and communicating with computer machines or Neuro-locomotive disabilitic patient are unable to use the mouse function as easily as normal people do. So this system interfaces between handicapped, Neuro- locomotive disabilitic patient and Computer. The gaze tracking system is based on light reflection theory. This theory uses non-imaging sensors such as photo-emitters and photo receivers and these provide accurate approximation point of gaze. To perform mouse operation by a handicapped using his eyes, we require IR (light) sensors. These are embedded on eye touch goggles, so that they can capture the movements of eyeball. Data acquisition system is used to gather and preprocess the data to plot the new position of mouse on screen. In this paper we used a method to improve the eye gaze tracking system so that it can track accurate eye gaze positions. The paper consists of different sections, like hardware and software used, proposed system etc.

\section{LITERATURE SURVEY}

In this section, we give an overview of methods used in gaze tracking research and also give the feature based and appearance based visible gaze tracking method. The main aim of gaze tracking is finding the accurate gaze position and gaze direction. There is a popular technique to solve this problem is electro-occulography. In this technique the sensors are used. i.e. electrode is for collecting the information about position of eyeball. In early eye tracking researches video-based eye tracking system was used for gaze movement, In those system the number of cameras are used for sampling, the eye proximity varies according to accuracy of the system.For video eye tracking system in order to reach better accuracy level higher resolution camera might be preferred, which increases cost of system. In other technique more than one computers were used one is for collecting and processing the data and other for actual movements to be done which could cost higher. Here are some of those methods.

Paper [10]:

1) Light-reflection based system.

2) Uses photo diodes or photo transistors.

3) A micro-controller based data acquisition system developed to accurately sample and digitize the analog sensor outputs

4) Relatively low complexity than camera based systems.

Paper [9]:

1) Camera based system for desktops.

2) Easy blink detection with less errors.

3) For accuracy needs high resolution of input image.

4) Inevitable and unintentional user head translation or rotation does not give proper results.

5) Free head motion remains a challenge.

Paper [2]:

1) Two computer system. Vision computer and user computer.

2) Visual tracking using live video of users gaze.

3) Different body parts are tracked like nose, mouth tracking, thumb tracking.

4) Suitable for children.

5) Visual tracking algorithm is used. 


\section{PROPOSED SYSTEM}

Our mission is to detect and track the human eyeball. When IR sensors emit the Infrared Light to the Human Eye, as we know human eye have two classified parts on their color intensity classification and those are: Sclera and Iris-Pupil. Every human eye sclera has a white color and mostly Irispupil is in black color. White color reflects light more as compared to the black where black color has strong absorbing power. The transmitter sensors transmit- light on eye and reflected light from eye parts is captured by receiver sensors mounted on goggles. The gathered data is sent to the computer system through software. Necessary calculations are done at the software using algorithms. When calculated data acquisition value in vector form is sent to the eye touch software then the software runs Gaze Approximation Algorithm i.e. least square algorithm. This Gaze Approximation algorithm is built on linear mapping and NonLinear mapping. After that using above mentioned algorithm cursor is moved according to movement of the eyeball. Working of proposed system is shown in figure below.

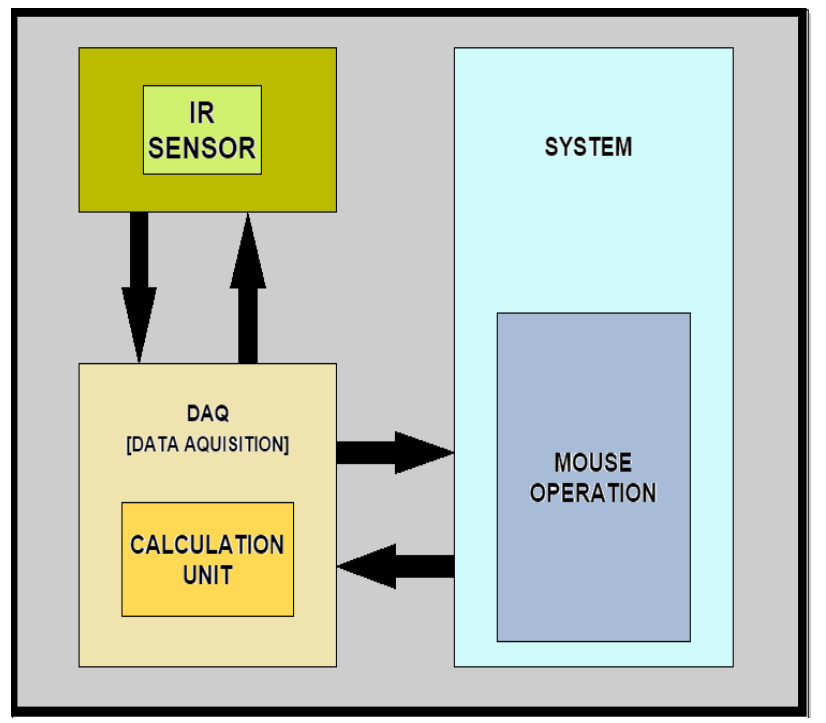

\section{SOFTWARE AND HARDWARE REQUIREMENTS}

In this section, we give the brief explanation of the software and hardware we are using in our system. This system consist three main parts. Two hardware are used and one software is used for the gaze estimation Eye Touch Goggles, Data Acquisition System, and Eye Touch Software.

A) Sensors: Light sensors are used to emit light and detect the infra-red light. Photo detectors and receivers are used. Photo detector and infrared emitter components are available in plastic or metal packages. A lens to improve radiant sensitivity or radiant intensity is used in most of the plastic devices. Detector chips are mounted on flat lead frame surfaces while lead frames for emitters have a silver plated reflector performing higher radiant intensity. Devices in metal packages are hermetically sealed, are released for extended operating temperature range and have small optical and mechanical tolerances.IR sensors used in this technique emit infra-red light of wavelength $950 \mathrm{~nm}$.

B) Eye Touch Goggles: To detect that there is movement Eye Touch Goggles are used. Eye touch goggles consists the IR sensors. As per the diagram below we mounted IR sensors on both the eye glasses. In the eye touch goggles there are two pairs of one transmitter and three receivers for both the eyes.
This reduces the number of IR sensors used in previous methods. IR sensor consists of a LED and a Photo diode to emit and detect the light waves. Transmitter emits the ultraviolet, infrared light whereas receiver detects. Eye touch goggle is connected to the data acquisition unit over the parallel communication cable.

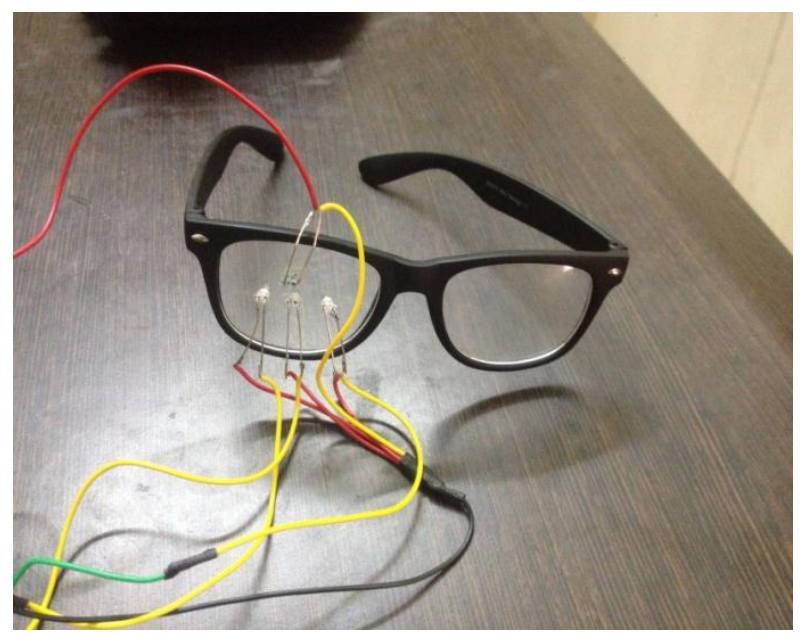

C) Accelerometer: Accelerometer is a device used to perform vertical movement of mouse pointer. It is electromechanical device and it is used to measure the acceleration forces. A dynamic accelerometer can measure gravitational pull which is used to determine the angle. Angle at which the device is tilted with respect to the Earth. It works by sensing the amount of acceleration. Accelerometers are used in tablet computers and also digital cameras so that image on screen can always be displayed upright. Single model of accelerometer are available to detect magnitude and direction of the proper acceleration.

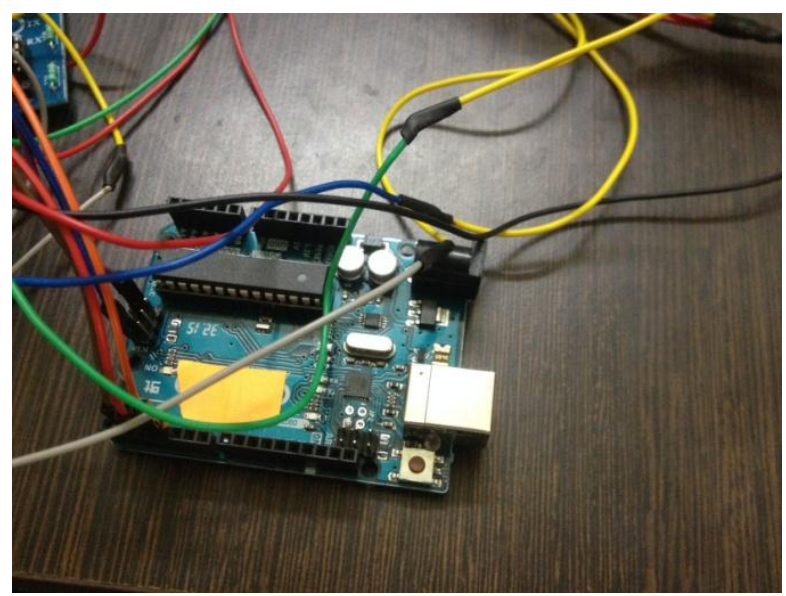

D) Data Acquisition Device: Data Acquisition Device (DAQ)is basically micro-controller based embedded system. The DAQ consist of 3 parts I/O system, Host Controlling system and Software. The main tasks of DAQ is as follows

1) Supplying a regular power supply to the goggle sensors.

2) Collecting analog signal from goggle sensors and sending that digital value to micro-controller to get values in vector form.

3) Sending digital values in vector form to the pc running software. 
E) Gaze Estimation Software: Gaze Estimation system is the software that runs on pc. The data sent by Data acquisition is collected by the gaze estimation software. This software listens to DAQ system and operates major tasks based on the information provided by gaze estimation

Software. These tasks performs some important functions as follows:

1) Gaze Estimation software collects data from DAQ which is in digital nature and process it.

2) It uses the least square algorithm for the gaze estimation then finds the position of cursor.

3) Interface is provide to the user.

4) By using pattern classification algorithm it performs major gaze operations like left click, right click, and double click.

\section{MOUSE OPERATIONS}

Clicking operations of mouse are based on the Human Eye wink. The classification algorithm determines the User's current position When Sensor recognizes the Eye from shorter distance. Then the click operation is performed.

a) RIGHT CLICK: When Right Eye is blinked then it performs Right click operation of mouse.

b) LEFT CLICK: As same as that of the left Click, when left eye is blinked left click will be performed.

c) CURSOR MOVEMENT: Whenever the eye gaze is relocated from one point to the another, position of cursor will move according to the gaze.

d) DOUBLE CLICK: When both eyes are blinked twice with a very short delay then double click operation can be performed.

To avoid collisions and for better performance there is no operation for simultaneous single blink of both eyes.

\section{IMPLEMENTATION}

Eye touch system uses sensors to detect the event. Sensors are based on light reflectance theory. The reflected light from the eye ball is more from white regions as that of iris.

But the difference almost linearly changes as the iris starts moving towards or away from a specific sensor, corresponding to left, right, up, or down eye movements. Before actual practical implementation, system training by user should be done. Implementation consists three parts:-

Implementation consists three parts:-

1) Data Collection and Preprocessing.

2) Click Classification.

3) Calibration

1. Data Collection and Preprocessing: While the data collection, user must steadily face the monitor and perform gazing and blink operations. User should train himself for better accuracy and smooth working of system before actual implementation. After each sampling point and blinking gesture, readings are collected from the sensor mounted on goggles.

2. Event Detection: when Data related to eye movements i.e., the sensor values are gathered, system uses a classification algorithm. This is algorithm helps to find out the current user event, which can be either left click, right click, double click, or gaze.

3. Calibration: After the classification algorithm determines that the current event done by user is gaze, eye touch system uses a gaze estimation algorithm. Gaze estimation algorithm is used to compute new cursor positions in real-time. If the user event is blinking then the according blinking operations will be performed on the screen and similarly other events will be performed as right click on right eye blinking and left click on left eye blink. Also Left movements on moving eyeball to left side and right side movements of cursor on moving the eyeball to right side can be performed.

\section{SIMULATION AND RESULTS}

The below graphs shows the some sample readings of the sensors noted. These readings are measured by five sensors. Sensor gives analog values. Transmitter transmits light to the eye. The light reflected from surface of eyeball is captured by receivers after reflection from eyeball. Three bars show the center, right and left positions detected of eyeball by the respected sensors.

Chart denotes the values captured by sensors when iris is at different positions left, right or middle.

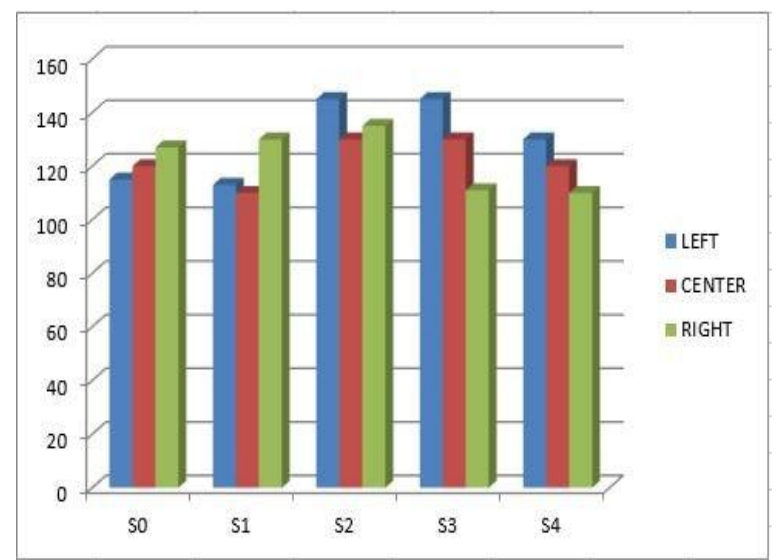

\section{CONCLUSION}

The mouse pointer through eye gaze detection system based on portable and low price components in wearable form is put forward in this paper. It will be implemented for gaze approximation and blink identification for click actions. Elimination of regular video camera and avoiding corresponding high estimation cost can be considered as an advantage of the proposed system. The output of these experiments indicated that eye gaze system results assured user interface alternative in those cases where controlling only by hand may be difficult. This gaze tracking system offers a capable accuracy under variable conditions like lighting and consumption problems. In previous systems, calculation of users gaze with image processing algorithms required more computational power and hence quick battery loss. On other side, with a similar approach to Eye Gaze, this problem can be solved with efficient and less effort manner. A restriction of the previous system was about being unable to move the head freely in its current form. Over past systems Idea of using goggles for gaze detection becomes a big advantage in head mounted displays. Head mounted displays are those where the vision is displayed on a pair of glasses, such that the display and the head move together. In such systems, a natural eye tracking system can be achieved with the inclusion of the mounted sensors. 


\section{SCOPE AND FUTURE WORK}

There is a big scope to make this system more user friendly. A lot work can be done on the goggles and sensor positions mounted on that goggle. Among the few approaches to eye tracking, Eye Touch system can become an alternative solution for mobile systems. This system can also be a very handful interface for those who need to deal with additional controls while doing their actual work. Eye Touch can be a solution for novel and efficient user interfaces for various applications, such as vehicle and wheelchair control for disabled. In the future, one can integrate the Eye Touch into a wearable computer having an HMD(Head Mounted Displays) rather than using the system with a desktop computer.

\section{ACKNOWLEDGMENTS}

It is our pleasure to acknowledge sense of gratitude to all those who helped us in making this project a reality. We thank our Guide Prof. S. R. Rathi for her exemplary guidance, monitoring and constant encouragement throughout the course of this paper. The blessing, help and guidance given by her shall time to time carry us a long way in the journey of life which we are about to embark. We are also thankful to Dr. S. R. Sakhare for providing us the required facilities and helping us while carrying out this project work. We are grateful for their cooperation during the period of our project

\section{REFERENCES}

[1] A. E. Kaufman, A. Bandopadhay, and B. D. Shaviv, An eye tracking computer user interface, in Proc. IEEE Symp. Res. Frontiers Virtual Reality, San Jose, CA, USA, Oct. 1993, pp. 120121.

[2] IEEE TRANSACTIONS ON NEURAL SYSTEMS AND REHABILITATION ENGINEERING, VOL. 10, NO. 1, MARCH 2002 The Camera Mouse: Visual Tracking of Body Features to Provide Computer Access for People With Severe Disabilities.

[3] D. W. Hansen and Q. Ji, In the eye of beholder: A survey of models for eyes and gaze, IEEE Trans.Pattern Anal. Mach. Intell., vol. 32, no.3, pp.478500, Mar. 2010.

[4] A. Plotkin, O. Shafrir, E. Paperno, and D. M. Kaplan, Magnetic eye tracking: A new approach employing a planar transmitter, IEEE Trans.Biomedical Eng., vol.57, no.5, pp. 12091215, May 2010.

[5] 2013 IEEE International Conference on Consumer Electronics (ICCE) A Novel Iris Center Localization
Method Based on the Spherical Eyeball Rotation Model for Human-Device Interaction.

[6] 2013 IEEE Transactions on Neural Systems and Rehabilitation Engineering A Nonlinear Model for Mouse Pointing Task Movement Time Analysis Based on Both System and Human Effects.

[7] IEEE Transactions on Consumer Electronics, Vol. 59, No. 2, May 2013 Eyeball Model-based Iris Center Localization for Visible Image-based Eye-Gaze Tracking Systems.

[8] IEEE TRANSACTIONS ON NEURAL SYSTEMS AND REHABILITATION ENGINEERING, VOL. 21, NO. 6, NOVEMBER 2013 A Dual-Mode Human Computer Interface Combining Speech and Tongue Motion for People with Severe Disabilities.

[9] IEEE TRANSACTIONS ON PATTERN ANALYSIS AND MACHINE INTELLIGENCE, VOL. 36, NO. 10, OCTOBER 2014 Adaptive Linear Regression for Appearance-Based Gaze Estimation.

[10] IEEE TRANSACTIONS ON CYBERNETICS, VOL. 44, NO. 2, FEBRUARY 2014 A Low-Computational Approach on Gaze Estimation With Eye Touch System.

[11] S.-W. Shih and J. Liu, A novel approach to 3-D gaze tracking using stereo cameras, IEEE Trans. Syst., Man, Cybern., vol. 34, no. 1, pp.234245, Feb. 2004.

[12] K. Talmi and J. Liu, Eye and gaze tracking for visually controlled interactive stereoscopic displays, Signal Process: Image Comm., vol.14, no. 10, pp. 799810, 1999.

[13] D. Beymer and M. Flickner, Eye gaze tracking using an active stereo head, in Proc. IEEE Conf. Computer Vision Pattern Recogn., vol. II.Jun. 2003, pp. 451458.

[14] S. C. Kim, M. Sked, and Q. Ji, Non-intrusive eye gaze tracking under natural head movements, in Proc. IEEE Eng. Med. Biol., San Francisco, CA, USA, Sep. 15, 2004, pp. 22712274.

[15] Y. Lin, Y. Chao, C. Lin, and J. Chen, Webcam mouse using face and eye tracking in various illumination environments, in Proc. IEEE Eng.Med. Biol., Shanghai, China, Sep. 14, 2005, pp. 37383741. 